\title{
Thrombocytopenia in obstetric patients
}

\author{
Mahmoud Alkholany, M.B.B.Ch, M.Sc. , FRCA. Clinical fellow of Anaesthesia, Central \\ Manchester university hospitals NHS foundation trust, U.K. Mohamed Mahmoud, \\ M.B.B.Ch, M.D, FRCA, Speciality trainee of Anaesthetics, North west deanery , U.K.
}

\section{Background:}

Safe conduct of Neuroaxial blockade mandates the presence of normal coagulation and platelet count and function to avoid serious complications such as epidural haematoma which can have devastating neurologic consequences. Obstetric patient population represents a cohort of patients with high frequency use of neuroaxial techniques for analgesia (lumbar epidural) and/or anaesthesia (spinal) which proved to be effective, superior and safer than use of GA. Despite this, presenting with low platelet count always constitutes a debate about which platelet count level is safer, whether the same level applies for spinal and epidural, whether the experience of the operator constitutes a factor in the specification of this safe level in addition to lack of high quality evidence.

\section{Methods:}

A retrospective audit, studied a number of obstetric patients who presented with thrombocytopenia during pregnancy in St Mary's hospital , Central Manchester university hospitals, UK. We reviewed the type of anaesthesia/analgesia the patients received with respect to their platelet count at time of presentation. We reviewed whether their anaesthesia/analgesia followed the agreed joint care plan formulated during antenatal care which doesn't recommend neuroaxial techniques with platelet count below $80,000 / \mathrm{ml}$. We also reviewed if there was any increase in the rate of GA as a consequence of following the joint care plan.
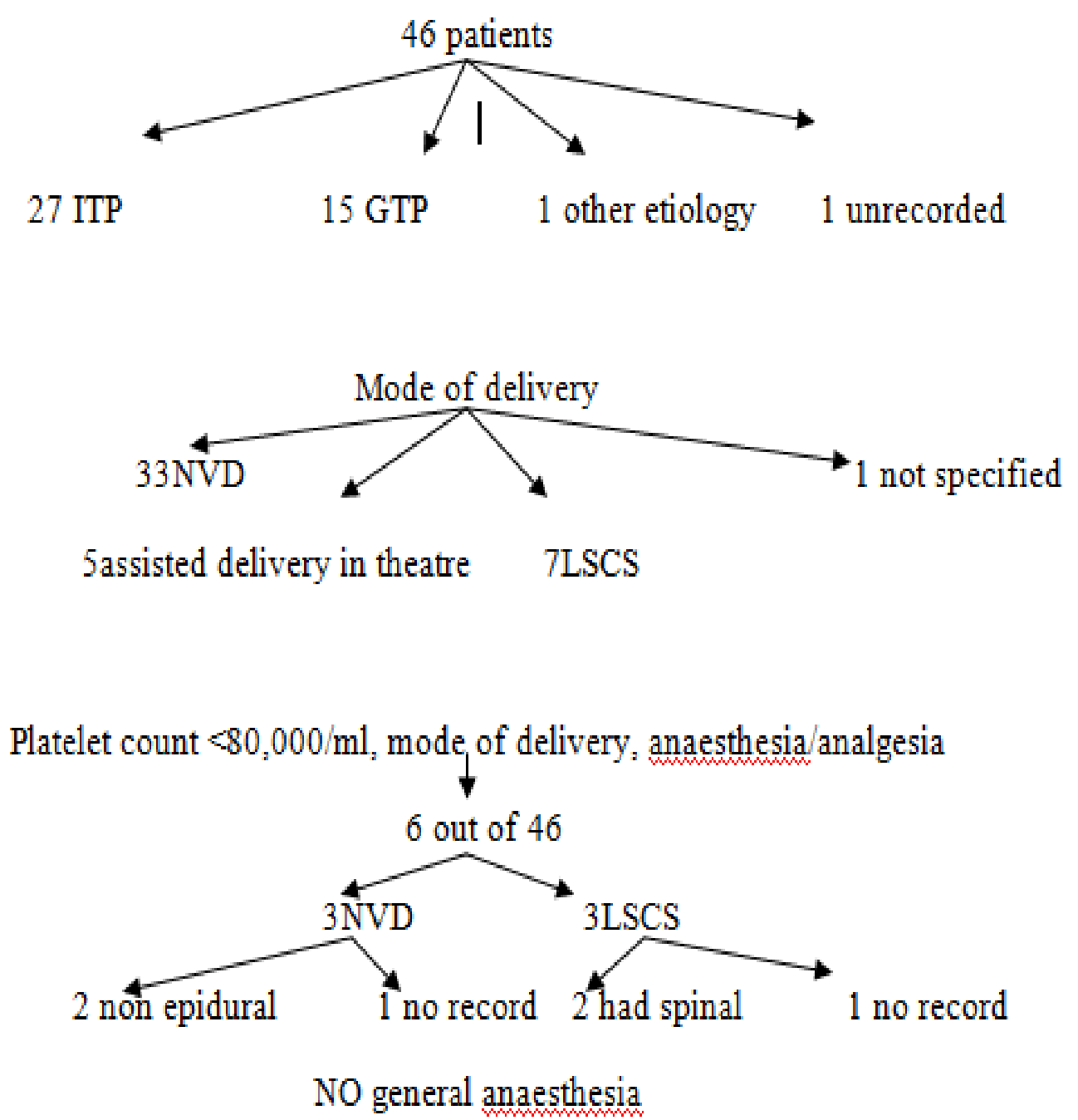

Variable EBL with no consistent correlation with platelet count or mode of delivery
Results:

1. 46 Patients studied in total.

2. 27 had ITP, 15 had GTP, 1 had other reason for low platelet count and 3 no recorded etiology.

3. 6 patients had platelet count equal or below $80,000 / \mathrm{ml}$ at time of delivery. 3 of them had LSCS for different reasons and 3 had NVD. In LSCS group, 2patients had spinal, one with platelet count $80,000 / \mathrm{ml}$ while the other $66,000 / \mathrm{ml}$, and the third didn't have recorded mode of anaesthesia. In NVD group, 2 had non neuroaxial analgesia, and the third one didn't have recorded mode of analgesia.

4. No patient received GA.

5. $100 \%$ had care plan in situ and seen in anaesthetic clinic antenataly. 2 patients had deviation from recommendation receiving spinal with platelet count $66,000 / \mathrm{ml}$ and $80,000 / \mathrm{ml}$ respectively, no complications recorded.

6. No transfusion of blood products was recorded.

\section{Conclusion:}

The decision to administer safe neuroaxial blockcade to obstetric patients is more complex than just a specific safe platelet count. It needs more evidence based decision making because current evidence depends more on expert opinion.

\section{Recommendations:}

1. Reaudit cycle is much needed, to be in line with AAGBI guidance on safe conduct of neuroaxial block in obstetric patients and extrapolate evidence from NAP 3 project as well.

2. Redefine standards to be met in terms of anaesthesia/analgesia care of obstetric patients with low platelet count.

3. Liaising with Hematology service to redefine and standardise safe platelet count level for performance of neuroaxial block in obstetric patients.

4. Encourage use of POC testing such as TEG to test for appropriateness of platelet function for performance of neuroaxial block in patients presenting with low platelet count.

5. More vigilance with recording details of care of obstetric patients with low platelet count which helps to tail plans for similar patients' cohort based on complication rate.

\section{References:}

1. Regional anaesthesia and patients with abnormalities of coagulation: The association of anaesthetists of great Britain and Ireland, the obstetric anaesthetists' association and regional anaesthesia UK. November 2013.

2. NAP 3: National Audit of Major Complications of Central Neuraxial Block in the United Kingdom 